\title{
Conditioned place preference for social interaction in rats: contribution of sensory components
}

\author{
Kai Kummer, Sabine Klement, Vincent Eggart, Michael J. Mayr, Alois Saria and Gerald Zernig*
}

Experimental Psychiatry Unit, Center of Psychiatry and Psychotherapy, Innsbruck Medical University, Innsbruck, Austria

Edited by:

Rainer Spanagel, Central Institute of

Mental Health, Germany

Reviewed by:

Osborne F. Almeida, University of

Minho, Portugal

Eduardo Butelman, Rockefeller

University, USA

\section{*Correspondence.}

Gerald Zernig, Experimental

Psychiatry Unit, Center of Psychiatry

and Psychotherapy, Innsbruck

Medical University, Innrain 66 a,

A-6020 Innsbruck, Austria.

e-mail: gerald.zernig@i-med.ac.at
A main challenge in the therapy of drug dependent individuals is to help them reactivate interest in non-drug-associated activities. We previously developed a rat experimental model based on the conditioned place preference (CPP) paradigm in which only four 15-min episodes of social interaction with a gender- and weight-matched male Sprague Dawley rat (1) reversed CPP from cocaine to social interaction despite continuing cocaine training and (2) prevented the reinstatement of cocaine CPP. In the present study, we investigated which of the sensory modalities of the composite stimulus "social interaction" contributes most to the rats' preference for it. If touch was limited by steel bars spaced at a distance of $2 \mathrm{~cm}$ and running across the whole length of a partitioning, CPP was still acquired, albeit to a lesser degree. If both rats were placed on the same side of a partitioning, rats did not develop CPP for social interaction. Thus, decreasing the available area for social interaction from 750 to $375 \mathrm{~cm}^{2}$ prevented the acquisition of CPP to social interaction despite the fact that animals could touch each other more intensely than through the bars of the partitioning. When touch was fully restricted by a glass screen dividing the conditioning chambers, and the only sensory modalities left were visual and olfactory cues, place preference shifted to place aversion. Overall, our findings indicate that the major rewarding sensory component of the composite stimulus "social interaction" is touch (taction).

Keywords: social interaction, sensory modalities, conditioned place preference, substance-use disorder, cocaine, touch, social stress, olfaction

\section{INTRODUCTION}

In the therapy of drug dependent individuals, it is a major goal to restructure the addict's life away from the drug of abuse toward non-drug-associated activities. For that strong alternative incentives are needed. Many studies report the worsening effects of social interaction on drug taking behavior. Social stress has been found to cause enhanced cocaine self-administration in rats and rhesus monkeys (Morgan et al., 2002; Miczek et al., 2011). In contrast to the deleterious effect of antagonistic stressful social interaction, Fritz et al. (2011) have recently shown in Sprague Dawley rats that social interaction, offered as four 15 -min episodes with a genderand weight-matched male conspecific is not only able to reverse conditioned place preference (CPP) from cocaine to social interaction despite continuing cocaine training, but even to prevent the reinstatement of cocaine CPP. This kind of dyadic social interaction may be similar to the psychotherapeutic situation, which, in combination with pharmacotherapy, has been shown to be a promising treatment (Carroll et al., 1998; Zernig et al., 2000) for substance dependence. It should be mentioned that many psychiatric disorders apart from substance dependence (e.g., autism, Rett syndrome, major depression, dysthymia, social anxiety disorder, generalized anxiety disorder, or social anhedonia in schizophrenia) find deficits in social reward not only concerning the interaction with health-care providers but during social interactions with family members, friends, and other social partners outside the health-care system. Therefore, investigating the neurobiological basis of this phenomenon seems of broader clinical importance.
The composite stimulus "social interaction" comprises a wide range of different sensory modalities, i.e., sight (vision), hearing (audition), smell (olfaction), taste (gustation), touch (taction), and, in the animal experimental setting described below, the flutter/vibration of the floor caused by the social interaction partner moving in close proximity (Squire et al., 2008). Visual cues are important for interaction among rat pups in food location (Galef and Clark, 1971), and rat's 50-kHz ultrasonic vocalizations induce approach behavior, whereas $22-\mathrm{kHz}$ signals lead to behavioral inhibition (Woehr and Schwarting, 2007). Rats are also able to discriminate between odors of submissive and dominant animals (Eisenberg and Kleiman, 1972). Further, gustatory cues on a demonstrator rat's breath affect the social transmission of food preference (Laland and Plotkin, 1993). In young rats, furry or soft tactile stimulation similar to litter huddling provides contact comfort, which can be used as an innate reinforcer (Alberts, 1978).

Inspired by the abovementioned findings and the likely complexity of interactions between the different sensory modalities, we investigated the extent to which these individual modalities might contribute to the composite stimulus 'social interaction'.

\section{MATERIALS AND METHODS ANIMALS}

Male Sprague Dawley rats $(n=85)$ were obtained from the Research Institute of Laboratory Animal Breeding of the Medical University Vienna (Himberg, Austria) and had to weigh 
150-250 g [corresponding to an age of 6-8 weeks, which can be considered early adulthood (Spear, 2000)] to be advanced to the CPP experiments. All animals were housed in groups of six rats until seven days before the start of the behavioral experiments, from which time on they were singly housed at a constant room temperature of $24^{\circ} \mathrm{C}$ and had ad libitum access to tap water and pelleted chow (Tagger, Austria). The rats were kept at a $12 \mathrm{~h}$ light/dark cycle with the lights on from $0800 \mathrm{~h}$ to $2000 \mathrm{~h}$. Experiments were conducted during the light period of the cycle.

The animals used in this study were cared for in accordance with the guidelines of the National Institutes of Health Animal Care and Use Program and the NIDA-IRP Animal Program, and the present experiments were approved by the Austrian National Animal Experiment Ethics Committee (permit number BMWF66.011/0019-II/10b/2010).

\section{CONDITIONED PLACE PREFERENCE APPARATUS}

Conditioning was conducted in a custom-made three-chamber apparatus $(64 \mathrm{~cm}$ wide $\times 32 \mathrm{~cm}$ deep $\times 31 \mathrm{~cm}$ high $)$ made of unplasticized polyvinyl chloride (uPVC). The middle (neutral) compartment $(10 \times 30 \times 30 \mathrm{~cm})$ had white walls and a white floor. Two doorways led to the two conditioning compartments $(25 \times 30 \times 30 \mathrm{~cm}$ each $)$ with walls showing either vertical or horizontal black-and-white stripes of the same overall brightness and with stainless steel floors containing either 168 holes (diameter $0.5 \mathrm{~cm})$ or 56 slits $(4.2 \times 0.2 \mathrm{~cm}$ each $)$. Time spent in each compartment was recorded with a video camera and analyzed offline with hand timers. After every single rat, the apparatus was cleaned with a $70 \%$ camphorated ethanol solution.

\section{PLACE CONDITIONING PROCEDURE}

For the acquisition of CPP to social interaction, the conditioning procedure comprised a pretest session on day one, eight consecutive training days (alternate-day-design, one training session per day, a total of four training sessions each for social interaction or saline), and a CPP test on day 10. To emphasize, pretest-, training-, and CPP test session lengths were of equal duration, i.e., $15 \mathrm{~min}=900 \mathrm{~s}$. Preference for any of the two conditioning chambers was declared if the rat spent more time in one of the conditioning chambers during pretest. The initially non-preferred chamber was subsequently paired with social interaction.

Social interaction consisted of a 15-min episode in one of the CPP compartments with a weight- and gender-matched conspecific immediately after an intraperitoneal (i.p.) injection of $1 \mathrm{ml} / \mathrm{kg}$ saline to control for possible handling- and i.p. injection effects. The conspecific remained the same dyadic partner for a total of four episodes. In the saline treatment condition, the same rat received the i.p. saline injection followed by solitary confinement in the other conditioning chamber. All experiments were performed using halogen white light (20 Watt) and radiogenerated white noise. Sensory components were controlled by introducing either uPVC insertions (experiments "social interaction" and "social interaction with weight difference"), uPVC insertions with steel bars on the bottom area running horizontally through the whole length (four bars, $2 \mathrm{~cm}$ apart; experiments "social interaction across bars" and "social interaction in a confined space"), uPVC insertions with holes (diameter $2 \mathrm{~mm}$; experiment "olfaction only") or a glass screen (experiment "vision, audition, olfaction and vibration") into the conditioning chamber.

\section{STATISTICAL ANALYSES}

All results are presented as group means \pm SEM. Behavioral results were analyzed using one-factor (time spent in the respective CPP chamber) analyses of variance (ANOVA) followed by post hoc comparisons using Tukey's range test and Pearson's correlation. Differences were considered significant at $p<0.05$. All statistical analyses were performed with Prism ${ }^{\circledR} 4$ (www.graphpad.com).

\section{RESULTS}

\section{COMPOSITE STIMULUS "SOCIAL INTERACTION"}

Social interaction within the confines of the CPP apparatus produced robust place preference, as evidenced by a significant increase in time spent in the interaction-paired compartment compared to the time spent in the saline-paired compartment (Figure 1A). One-factor ANOVA yielded a significant effect of the treatment factor (social interaction [int] vs. neutral [neu] vs. saline [sal]; $n=14, F=11.56, p<0.0001$ ), post hoc comparison showed a significant preference for social interaction over saline $(p<0.001)$.

\section{SOCIAL INTERACTION ACROSS BARS}

When the ability to touch the interaction partner was restricted by an opaque partitioning with steel bars running horizontally through the whole length, CPP was still acquired, albeit to a lesser degree (Figure 1B). One-factor ANOVA yielded a significant effect of the treatment factor (int vs. neu vs. sal; $n=21$, $F=26.92, p=0.0001)$, post hoc comparison showed a significant preference for social interaction over saline $(p<0.05)$.

\section{SOCIAL INTERACTION IN A CONFINED SPACE}

If both rats were placed on the same side of the barred partitioning, thus decreasing the available area for social interaction from 750 to $375 \mathrm{~cm}^{2}$, CPP did not develop, as shown in almost equal times spent in the interaction- and saline-paired compartments (Figure 1C). Although one-factor ANOVA yielded a significant effect of treatment (int vs. neu vs. sal; $n=15, F=8.74$, $p=0.0007)$, post hoc comparison did not show a significant preference for social interaction over saline $(p>0.05)$.

\section{VISION, AUDITION, OLFACTION AND VIBRATION}

When a glass screen was introduced into the conditioning chambers to fully inhibit tactile interaction, animals did acquire conditioned place aversion (CPA), i.e., they spent more time in the saline-paired compartment compared to the interaction-paired compartment (Figure 1D). One-factor ANOVA yielded a significant effect of the treatment factor (int vs. neu vs. sal; $n=5$, $F=5.99, p=0.016$ ), post hoc comparison showed a significant preference for saline over social interaction.

\section{OLFACTION ONLY}

An isolated olfactory stimulus was presented by introducing an opaque screen with perforations to the conditioning chambers, and placing a bowl with another rat's bedding behind it. This 


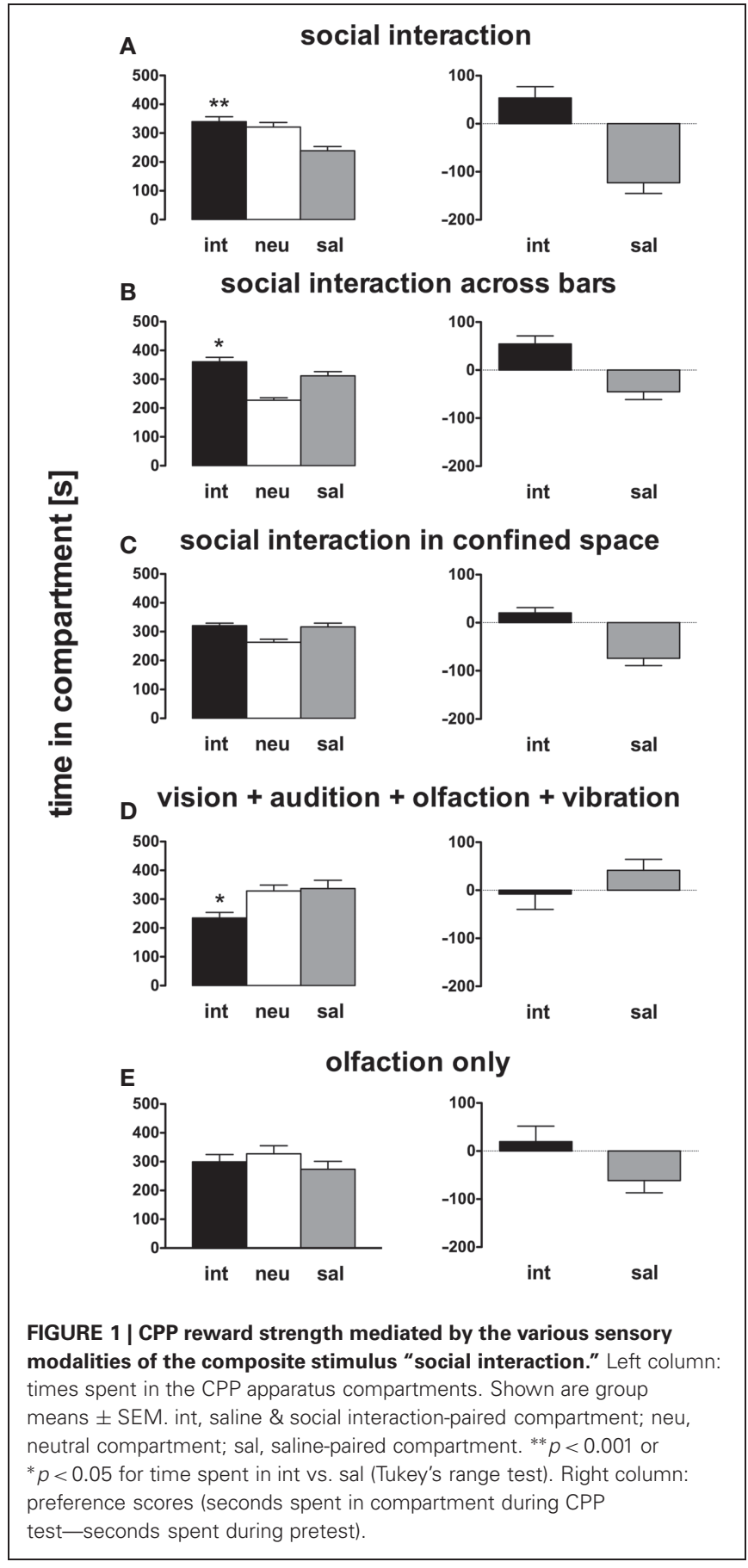

olfactory contact did not elicit CPP, as shown in almost equal times spent in the interaction- and saline-paired compartments (Figure 1E). One-factor ANOVA yielded no significant effect of treatment (int vs. neu vs. sal; $n=7, F=0.99, p<0.39$ ), post hoc comparison did not show a significant preference for any combination $(p>0.05)$.

\section{SOCIAL INTERACTION WITH WEIGHT DIFFERENCE}

In this last experiment the test rats were paired for social interaction with stimulus rats of up to twice the weight of the respective test rat. For test rats $(n=11)$, there was a significant negative correlation between weight difference and time spent in the interaction-paired compartment (Figure 2A; $r=-0.74$, $p=0.0093$ ), a significant positive correlation between weight difference and time spent in the neutral compartment $(r=0.84$, $p=0.0013)$ and no significant correlation between weight difference and time spent in the saline-paired compartment $(r=-0.53, p=0.092)$. For stimulus rats $(n=12)$, there were no significant correlations between weight difference and times spent in the respective compartments (int: Figure 2B, $r=-0.29$, $p=0.36$; neu: $r=0.035, p=0.91$; sal: $r=0.25, p=0.44$ ).

\section{DISCUSSION}

Confirming findings obtained by the previous generation of experimenters in our group (Fritz et al., 2011), we could show that only four 15-min episodes of social interaction with a weight-matched (and, as per gross observation, non-dominant) conspecific of the same (male) gender produced CPP for social interaction (Figure 1A). This kind of social interaction had previously been shown to be able to reverse cocaine CPP and inhibit cocaine-induced reinstatement of cocaine CPP (Fritz et al., 2011). The present findings indicate that the most rewarding component of the composite stimulus "social interaction" is touch (taction). Even if a partitioning was introduced into the conditioning chamber, so that the rats could only touch each other with their snouts, forepaws, and tails through steel bars spaced $2 \mathrm{~cm}$ apart, CPP for social interaction was still acquired, although in a less pronounced manner (Figure 1B). This finding strongly indicates that the intensity of touch (taction) determines the rewarding properties of social interaction to a considerable degree. When touch was fully restricted by a glass screen dividing the conditioning chambers, and the only sensory modalities left were visual, auditory, vibrational and olfactory cues, CPP shifted to CPA (Figure 1D). Thus, it seems that in rats touch (taction) contributes most to the composite stimulus "social interaction." Surprisingly for a species which has traditionally been endowed by conventional wisdom with great powers of smell [in contrast to humans, which have only about 350 functional olfactory receptor genes, rodents have about 1300 functional genes (Shepherd, 2004)] simply smelling the bedding of another rat across an opaque dividing screen (to eliminate acoustic interaction) did not produce place preference. To see, smell, and hear an interaction partner but not being able to touch him seems not to be rewarding at all for an early adult male Sprague Dawley rat.

In some (Covington et al., 2008) resident-intruder models, i.e., in experimental approaches investigating social interaction of the hostile type, a weight difference between the tested rat (the smaller rat) and the hostile social interaction partner (the bigger rat) is introduced, in all likelihood to increase the aversiveness of the hostile interaction. We, therefore, systematically varied the weight of the social interaction partner and found, even in a social interaction of the friendly type, that the larger the social interaction partner was the less rewarding the interaction became for the smaller $\left(r^{2}=0.55\right.$; Figure 2A) but not the bigger rat $\left(r^{2}=0.09\right.$; Figure 2B). Under those conditions, the neutral chamber, which was not paired with any contextual cues, seemed to have become more attractive than the saline-paired chamber, which 


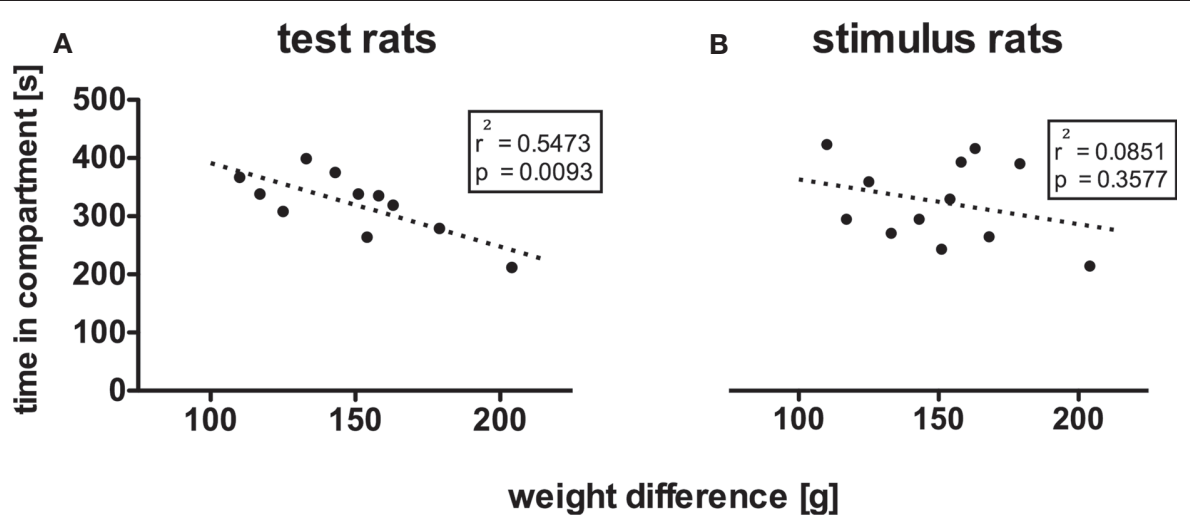

FIGURE 2 | Weight difference decreases social interaction CPP more in test rats than in stimulus rats. Pearson's correlation of weight difference and time spent in the interaction-paired chamber yielded a significant negative correlation $\left(r^{2}=0.5473, p=0.0093\right)$ for test rats (A), and no statistically significant correlation for stimulus rats (B).

the rats associated with handling necessary for i.p. injections and the saline injections themselves, before being put into the chamber. If one allows that saline injections might be aversive per se, and when the usually rewarding social interaction is becoming less so, the rats may have plausibly shifted their place preference to the neutral chamber, which may have also become preferred to the less attractive social interaction-associated conditioning chamber because the neutral chamber may have constituted a "more novel" environment [see Bardo et al. (1989) for noveltyinduced place preference]. It should be noted, however, that the neutral chamber was not completely novel to the animals: they had $15 \mathrm{~min}$ to explore it during the pretest, and were, through the glass doors, in full visual contact with the neutral chamber during all conditioning sessions. We would opine that the lack of any associated injection memories rendered the neutral chamber more attractive under the experimental conditions described above. It seems that with a weight difference of approximately $150 \mathrm{~g}$ (which equals $\sim 75 \%$ of the tested rats' weight which was, on average, $200 \mathrm{~g}$ ), the clear preference for the social interaction-paired chamber disappeared. This suggests that in social defeat experiments which use weight differences of only about $75 \mathrm{~g}$ [e.g., Covington and Miczek (2005)], other factors must be responsible for the offensive aggressive interactions, e.g., that the test rats intruded into the social interaction partner's compounds which were previously housed by a dedicated female. For the social interaction partners which weighed around $350 \mathrm{~g}$, there was neither an acquisition of social interaction CPP nor a significant correlation between the different chambers and the conspecifics' weight difference.

\section{REFERENCES}

Alberts, J. R. (1978). Huddling by rat pups: multisensory control of contact behavior. J. Comp. Physiol. Psychol. 92, 220-230.

Bardo, M. T., Neisewander, J. L., and Pierce, R. C. (1989). Novelty-induced place preference behavior in rats: effects of opiate and dopaminergic drugs. Pharmacol. Biochem. Behav. 32, 683-689.

Carroll, K. M., Nich, C., Ball, S. A., Mccance, E., and Rounsavile, B. J. (1998). Treatment of cocaine and alcohol dependence with psychotherapy and disulfiram. Addiction 93, 713-727.

Considering that social interaction is able to reverse cocaine $\mathrm{CPP}$ and inhibit cocaine-induced reinstatement of cocaine CPP (Fritz et al., 2011), the present findings suggest that "body-" (i.e., touch-) centered psychotherapeutic approaches would be more effective than psychotherapies that rely mostly on verbal interaction ("talking cures") for the treatment of substance-use disorders. The respective comparative clinical trials still have to be performed.

To go beyond this most obvious translational aspect of the present findings, it should be emphasized that social interaction is impaired in a variety of psychiatric disorders (e.g., autism, Rett syndrome, major depression, dysthymia, social anxiety disorder, generalized anxiety disorder, or social anhedonia in schizophrenia), making it worthwhile to investigate the behavioral neurobiology of social interaction in pathophysiological states, and also as a therapeutic intervention. Touch is the sensory component that governs social interaction in our experimental setting in the rat species, and is therefore a very valuable methodological focus and construct to understand social attachment if one keeps in mind that social interaction in humans most likely has other important components in addition or instead of taction.

\section{ACKNOWLEDGMENTS}

This work was supported by the Austrian Science Fund grants P18787-B05 (Gerald Zernig) and W1206-B18 (PhD program "Signal Processing in Neurons", Kai Kummer) and by the Verein fuer Experimentelle Psychiatrie, Psychotherapie und Pharmakologie (VEPPP).

Covington, H. E. 3rd, and Miczek, K. A. (2005). Intense cocaine selfadministration after episodic social defeat stress, but not after aggressive behavior: dissociation from corticosterone activation. Psychopharmacology 183, 331-340.

Covington, H. E. 3rd, Tropea, T. F., Rajadhyaksha, A. M., Kosofsky,
B. E., and Miczek, K. A. (2008). NMDA receptors in the rat VTA: a critical site for social stress to intensify cocaine taking. Psychopharmacology 197, 203-216.

Eisenberg, J. F., and Kleiman, D. G. (1972). Olfactory communication in mammals. Annu. Rev. Ecol. Syst. 3, 1-32. 
Fritz, M., El Rawas, R., Salti, A., Klement, S., Bardo, M. T., Kemmler, G., Dechant, G., Saria, A., and Zernig, G. (2011). Reversal of cocaine-conditioned place preference and mesocorticolimbic Zif268 expression by social interaction in rats. Addict. Biol. 16, 273-284.

Galef, B. G. Jr., and Clark, M. M. (1971). Social factors in the poison avoidance and feeding behavior of wild and domesticated rat pups. J. Comp. Physiol. Psychol. 75, 341-357.

Laland, K., and Plotkin, H. (1993). Social transmission of food preferences among Norway rats by marking of food sites and by gustatory contact. Anim. Learn. Behav. 21, 35-41.

Miczek, K. A., Nikulina, E. M., Shimamoto, A., and Covington,
H. E. 3rd (2011). Escalated or suppressed cocaine reward, tegmental BDNF, and accumbal dopamine caused by episodic versus continuous social stress in rats. J. Neurosci. 31, 9848-9857.

Morgan, D., Grant, K. A., Gage, H. D., Mach, R. H., Kaplan, J. R., Prioleau, O., Nader, S. H., Buchheimer, N., Ehrenkaufer, R. L., and Nader, M. A. (2002). Social dominance in monkeys: dopamine D2 receptors and cocaine selfadministration. Nat. Neurosci. 5, 169-174.

Shepherd, G. M. (2004). The human sense of smell: are we better than we think? PLoS Biol. 2, E146. doi: 10.1371/journal.pbio. 0020146

Spear, L. P. (2000). The adolescent brain and age-related behavioral manifes- tations. Neurosci. Biobehav. Rev. 24, 417-463.

Squire, L., Berg, D., Bloom, F., Du Lac, S., Ghosh, A., and Spitzer, N. (2008). Fundamental Neuroscience. Amsterdam: Elsevier.

Woehr, M., and Schwarting, R. K. (2007). Ultrasonic communication in rats: can playback of $50-\mathrm{kHz}$ calls induce approach behavior? PLoS One 2, e1365. doi: 10.1371/ journal.pone.0001365

Zernig, G., Saria, A., Kurz, M., and O'Malley, S. S. (2000). Handbook of Alcoholism. Boca Raton, FL: CRC Press.

Conflict of Interest Statement: The authors declare that the research was conducted in the absence of any commercial or financial relationships that could be construed as a potential conflict of interest.

Received: 05 September 2011; accepted: 14 November 2011; published online: 30 November 2011.

Citation: Kummer K, Klement S, Eggart V, Mayr MJ, Saria A and Zernig $G$ (2011) Conditioned place preference for social interaction in rats: contribution of sensory components. Front. Behav. Neurosci. 5:80. doi: 10.3389/ fnbeh.2011.00080

Copyright (c) 2011 Kummer, Klement, Eggart, Mayr, Saria and Zernig. This is an open-access article distributed under the terms of the Creative Commons Attribution Non Commercial License, which permits non-commercial use, distribution, and reproduction in other forums, provided the original authors and source are credited. 